\title{
Fotocatálisis/Adsorción para la Eliminación de Carbono Orgánico Total presente en Vapor Condensado de Cocción generado en el Tratamiento de Subproductos Avícolas
}

\author{
Andrés F. López-Vásquez*, Johana M. Franco-Macias, Erika J. Montes-Alba \\ Universidad Libre, Facultad de Ingeniería, Departamento de Ingeniería Ambiental, Bogotá, Colombia. \\ (e-mail: andresf.lopez@unilibrebog.edu.co; johanam.francom@unilibrebog.edu.co; \\ erikaj.montesa@unilibrebog.edu.co) \\ * autor a quien debe ser dirigida la correspondencia
}

Recibido Jun. 1, 2016; Aceptado Ago. 3, 2016; Versión final Sep. 12, 2016, Publicado Feb. 2017

\begin{abstract}
Resumen
Se evaluó la remoción de carbono orgánico total (COT) presente en un efluente generado por la industria de procesamiento de subproductos de origen animal (rendering), mediante fotocatálisis (foto-Fenton) y adsorción con carbón activado. El efluente considerado fue el vapor condensado de cocción cuya elevada carga contaminante, hace que tratamientos convencionales tales como los biológicos y químicos sean insuficientes o económicamente inviables. Para evaluar el efecto de las variables de proceso, concentración de $\mathrm{Fe}(\mathrm{II})$ y dosis de agente oxidante $\left(\mathrm{H}_{2} \mathrm{O}_{2}\right)$, se utilizó la metodología de superficie de respuesta. En el estudio de adsorción se consideró el efecto del $\mathrm{pH}$, dosis de adsorbente y tiempo de contacto. Para el tratamiento fotocatalítico se alcanzó una remoción del $69.2 \%$ de COT, mientras que para el proceso de adsorción fue del $77.9 \%$. Después de esto, se evaluó la remoción de COT de dos sistemas acoplados. Para uno de los sistemas, el efluente fue pre tratado mediante adsorción y sometido posteriormente al tratamiento fotocatalítico donde se alcanzó una degradación global del $91.9 \%$. Esta sinergia entre los procesos de adsorción y foto-Fenton, aparecen como una alternativa viable para el tratamiento de este tipo de efluentes industriales.
\end{abstract}

Palabras clave: adsorción; foto-Fenton; procesamiento de subproductos avícolas; sistema acoplado adsorción-fotocatálisis; vapor condensado de cocción

\section{Photocatalysis/Adsorption for Total Organic Carbon Removal present in Condensed Steam Cooking generated in Poultry By-products Treatment}

\begin{abstract}
In this paper the removal of total organic carbon (TOC) present in an effluent generated by the rendering industry was evaluated, by photocatalysis (photo-Fenton) and activated carbon adsorption. The effluent considered in the study was the condensed steam cooking that has high environmental burdens so conventional biological and chemical treatments are insufficient or are economically unviable. To evaluate the effect of process variables, concentration of $\mathrm{Fe}$ (II) and dose of oxidizing agent $\left(\mathrm{H}_{2} \mathrm{O}_{2}\right)$, the response surface methodology was used. In the adsorption study, the effect of $\mathrm{pH}$, adsorbent dose and contact time was considered. For photocatalytic treatment the TOC removal percentage obtained was $69.2 \%$, while for the adsorption process was $77.9 \%$. After that, the TOC removal of two coupled systems was evaluated. For one of the systems, the effluent was pretreated by adsorption and subsequently subjected to photocatalytic treatment, reaching an overall degradation of $91.9 \%$. This synergy between adsorption and photo-Fenton processes appears as a viable option for the treatment of this type of industrial effluents.
\end{abstract}

Keywords: adsorption; photo-Fenton; rendering; adsorption-photocatalysis coupled system; cooking condensed steam 


\section{INTRODUCCIÓN}

Existen técnicas de procesamiento que le dan valor agregado a los residuos generados en las plantas de procesamiento de subproductos avícolas, los cuales son aprovechados para transformarlos en materia prima para la elaboración de concentrado para animales. Este procedimiento se conoce con el nombre de "rendering" que comprende etapas de trituración, cocción, extracción de humedad y separación de grasa para obtener tres tipos de harina correspondientes a sangre, plumas y vísceras (Meeker, 2006). Esta técnica genera aguas residuales en cada uno de los procesos mencionados anteriormente las cuales experimentan problemas de espumación y altas concentraciones de grasa y sólidos en el vapor condensado (Sindit, 2006). Uno de estos efluentes es conocido como vapor condensado de cocción (VCC), que se caracteriza por poseer olor desagradable, elevada demanda química y biológica de oxígeno, ligera turbidez, pH alcalino y además contiene compuestos orgánicos condensables, altas concentraciones de amoniaco, grasa en aerosol y residuos sólidos provenientes del proceso de cocción. Debido a estas características, tanto los vapores de cocción como el vapor condensado, no se pueden verter en las fuentes naturales puesto que generaría un fuerte impacto ambiental sobre el recurso.

Algunas estrategias de remediación contemplan desde la filtración, sedimentación, aplicación de tratamientos biológicos, etc. hasta técnicas más complejas que eliminan contaminantes específicos con el fin de cumplir con límites de descarga los cuales no se alcanzan con tratamientos primarios y secundarios convencionales. Para el tratamiento del VCC, se han implementado tratamientos térmicos (corto tiempo) a elevadas temperaturas para destruir los compuestos orgánicos volátiles y descargar los gases tratados a la atmósfera. Otra alternativa es la dilución de corrientes que consiste en condensar el vapor de agua generado en el proceso de cocción y mezclarlo con los demás efluentes generados en el proceso pero por las características del VCC, se considera una opción inapropiada (Sindit, 2006).

Una opción tecnológica para el tratamiento de efluentes, es la combinación de dos o más técnicas con el fin de aumentar la eficiencia de remoción en los procesos de descontaminación. Recientemente, se han probado procesos avanzados de oxidación (Crini, 2006; Malato, 2004) para la remoción de carga contaminante presente en efluentes de la industria textil (colorantes) (Thinakaran, 2008; Gupta, 2004), compuestos orgánicos (Djilani et al., 2012; Delfin et al., 2009; López et al., 2016), productos farmacéuticos (Zhang et al., 2012) y metales pesados (Delfin et al., 2009; Sayed et al., 2005), en combinación con tratamientos primarios tales como la adsorción (Wei et al., 2008; Ho y McKay, 1999) en la que predomina el uso del carbón activado (Moreno et al., 2006; Moreno y Giraldo, 2008). El objetivo de acoplar estas técnicas ha sido aprovechar el efecto sinérgico de ambos tratamientos con el fin de mejorar la remoción de carga contaminante (Kumar y Bansal, 2010; Metiver et al., 2003) aunque en ocasiones, no se alcancen resultados satisfactorios (Shon et al., 2005; Kebir et al., 2011).

En este trabajo se evaluó el efecto sinérgico del acople de un tratamiento avanzado de oxidación (fotoFenton) y un proceso de adsorción (carbón activado natural) para la remoción de carga orgánica contaminante representada como carbono orgánico total (COT) presente en el vapor condensado de cocción generado durante el tratamiento de subproductos avícolas.

\section{METODOLOGÍA}

Se decriben los materiales, luego se explica la forma de remoción de COT presente en el VCC y finalmente se detalla la evaluación del acople de los tratamientos foto-Fenton y adsorción.

\section{Materiales}

Se tomó una muestra representativa de VCC directamente de un efluente residual de una industria local de "rendering" y no se le realizó tratamiento adicional. La caracterización física y química se realizó con base en los Métodos Normalizados para el Análisis de Aguas Potables y Residuales (APHA-AWWA y WPCF, 1992). Para las pruebas fotocatalíticas se utilizó $\mathrm{FeCl}_{3} \cdot 6 \mathrm{H}_{2} \mathrm{O}$ (Merck) y $\mathrm{H}_{2} \mathrm{O}_{2} 30 \% \mathrm{v} / \mathrm{v}$ (Merck) mientras que la adsorción se realizó con carbón activado (CA) como material adsorbente (obtenido a partir de un material orgánico generado durante el mismo proceso de rendering). Tanto para las pruebas fotocatalíticas como de adsorción, el ajuste del pH se realizó mediante adición de soluciones de $\mathrm{KOH} 3 \mathrm{M}$ (Merck) y HCl 3M (SigmaAldrich).

\section{Remoción de carbono orgánico total presente en el VCC.}

La evaluación del tratamiento fotocatalítico y de adsorción, se realizó mediante la determinación del porcentaje de remoción de COT. Para el proceso de foto-Fenton se evaluó el efecto de las variables de proceso mediante un diseño factorial $2^{\mathrm{k}}$ (cara centrada). Los niveles de los factores fueron 0.25-1.25 g Fe (II)/l y 1.0-3.0 $\mathrm{ml} \mathrm{H} \mathrm{O}_{2}$. La optimización de variables de proceso se realizó mediante la metodología de 
superficie de respuesta (Gutiérrez y De la Vara, 2008; Montgomery, 2004) a través del análisis estadístico desarrollado con el software Desing Expert 7.0 (Trial versión). Los experimentos fotocatalíticos se llevaron a cabo en un reactor fotoquímico (ACE-Glass) a pH 3.0 durante 120 min (la temperatura se mantuvo constante a $25^{\circ} \mathrm{C}$ ). El carbón activado $(\mathrm{CA})$ utilizado como material absorbente obtenido a partir del material orgánico mediante un proceso de calcinación simple bajo atmósfera oxidante, se lavó con agua destilada y deionizada y se secó en un horno a $80^{\circ} \mathrm{C}$ durante $24 \mathrm{~h}$. Este se caracterizó mediante microscopía electrónica de barrido (TESCAN-Vega 3) y espectroscopía infrarroja por transformada de Fourier (IR Prestige-21, Shimadzu). Para la evaluación del proceso de adsorción, se consideró el efecto de variables tales como $\mathrm{pH}$, dosis de adsorbente y tiempo de contacto. El proceso se realizó en modo discontinuo. La capacidad de adsorción y afinidad entre los sitios activos del adsorbente y los iones del soluto se analizó mediante los modelos de isoterma de Freundlich (Özer y Pirincci, 2006) y Langmuir (Al-Anber y Mohammed Abu, 2008) y se determinó la capacidad de adsorción para el sistema en fase liquida mediante la ecuación (1) (Azouaou et al., 2010).

$$
\mathrm{q}_{\mathrm{e}}=\frac{\mathrm{V}\left(\mathrm{C}_{0}-\mathrm{C}_{\mathrm{e}}\right)}{\mathrm{m}}
$$

donde $q_{e}$, es la cantidad de sorbato adsorbido en el equilibrio ( $\mathrm{mg}$ sorbato/g de peso seco de material adsorbente), $V$ corresponde al volumen de la solución de sorbato en contacto con el adsorbente, $C_{0}$ es la concentración inicial del sorbato $(\mathrm{mg} / \mathrm{l}), C_{e}$ es la concentración final o de equilibrio del sorbato en la solución (mg/l) y $m$ corresponde a la masa de adsorbente en seco añadido (g) (Li et al., 2012). Para determinar la velocidad con la que el sorbato fue removido de la solución acuosa (Yank, 2003), se utilizaron los modelos de pseudo primer orden (Awwad y Salem, 2011), pseudo segundo orden (Kumar, U y Bandyopadhyay, 2005) y difusión intraparticular (Ahmad et al., 2011).

\section{Evaluación del acople de los tratamientos foto-Fenton y adsorción.}

La sinergia entre los sistemas acoplados fotocatálisis/adsorción y adsorción/fotocatálisis se evaluó bajo las condiciones óptimas determinadas tanto para el proceso fotocatalítico como para la adsorción. Para tal fin, se determinó el porcentaje global de remoción de carbono orgánico total a la salida de los sistemas acoplados.

\section{RESULTADOS Y DISCUSIÓN}

Los resultados se presentan y discuten en tres subsecciones: caracterización del efluente, tratamiento foto catalítico (foto-fenton) y acople de los tratamientos de fotocatálisis y adsorción

\section{Caracterización del efluente}

Los resultados de la caracterización física y química del VCC se muestran en la Tabla 1. Con base en los valores establecidos en la normatividad local para vertimientos industriales, el VCC no cumple con los requisitos mínimos de calidad del efluente (Sindit, 2006; Ardila et al., 2012).

Tabla 1: Caracterización física y química del vapor condensado de cocción generado durante el proceso de rendering

\begin{tabular}{|l|c|}
\hline \multicolumn{1}{|c|}{ Parámetro } & Valor \\
\hline Potencial de hidrógeno $(\mathrm{pH})$ & $8.7-8.9$ \\
\hline Demanda biológica de oxígeno (DBO) & $400-671 \mathrm{ppm}$ \\
\hline Demanda química de oxígeno (DQO) & $1185.65-1924.89 \mathrm{ppm}$ \\
\hline Carbono orgánico total (TOC) & $235.5-405.9 \mathrm{ppm}$ \\
\hline Sólidos suspendidos totales (SST) & $0.60 \mathrm{~g} / \mathrm{L}$ \\
\hline Sólidos disueltos (SD) & $0.994 \mathrm{~g} / \mathrm{L}$ \\
\hline Sólidos totales (ST) & $1.60 \mathrm{~g} / / \mathrm{L}$ \\
\hline Turbiedad & $20.2 \mathrm{NTU}$ \\
\hline
\end{tabular}

Con base en lo anterior, el índice de biodegradabilidad del agua residual ( $\left.\mathrm{DBO}_{5} / \mathrm{DQO}\right)$ del VCC fue de 0.34 , lo que significa que es un efluente poco biodegradable (Ministerio de Ambiente y Desarrollo Sostenible, 2015) y un simple tratamiento biológico no sería suficiente para remover la carga orgánica contaminante presente en el vertimiento. Bajo las condiciones actuales, la descarga del vertimiento sin ningún tipo de tratamiento provocaría la pérdida de las cualidades organolépticas del medio receptor (olor, sabor, turbidez), lo que daría inicio o aceleraría los procesos de eutrofización (Ministerio de Ambiente y Desarrollo Sostenible, 2013). 


\section{Tratamiento fotocatalítico (foto-Fenton)}

El análisis de varianza indicó los parámetros más influyentes del proceso. Todos aquellos factores o interacciones entre ellos mismo que obtengan un valor de probabilidad $\mathrm{P}<0.05$ (última columna de la Tabla 2), son considerados como los más relevantes en el proceso para obtener la máxima remoción de la concentración COT de VCC en este caso, los factores A y B, fueron los más significativos. El análisis estadístico se muestra en la Tabla 2.

Tabla 2: Análisis de varianza para el proceso de fotocatálisis homogénea

\begin{tabular}{|c|c|c|c|c|c|}
\hline Fuente & Suma de cuadrados & g.l. & Cuadrado medio & Valor $F$ & P-Valor Probabilidad $>F$ \\
\hline Modelo & 942.36 & 5 & 188.47 & 77.55 & $<0.0001$ (significativo) \\
\hline A-Fe (II) & 612.67 & 1 & 612.67 & 252.11 & $<0.0001$ \\
\hline $\mathrm{B}-\mathrm{H}_{2} \mathrm{O}_{2}$ & 328.86 & 1 & 328.86 & 135.32 & $<0.0001$ \\
\hline$A B$ & 0.032 & 1 & 0.032 & 0.013 & 0.9126 \\
\hline$A^{2}$ & 0.76 & 1 & 0.76 & 0.31 & 0.5998 \\
\hline $\mathrm{B}^{2}$ & 0.18 & 1 & 0.18 & 0.074 & 0.7962 \\
\hline Residual & 12.15 & 5 & 2.43 & & \\
\hline Carencia de ajuste & 6.55 & 3 & 2.18 & 0.78 & 0.6039 (no significativo) \\
\hline Total & 954.51 & 10 & & & \\
\hline $\mathrm{R} 2$ & 0.9873 & & & & \\
\hline
\end{tabular}

De acuerdo al ajuste, se obtuvo una correlación (R2) de 98.73\%. Este valor explica el porcentaje de variables que afectan el porcentaje de remoción de COT (\% Rem. COT) y la diferencia de $1.27 \%$ muestra la minimización de las posibles causas de error. La ecuación que ajustó el modelo fue dada por (2):

$$
\text { \% Rem. COT: } 51.59-23.13 \times \mathrm{Fe}(\mathrm{II})+8.61 \times \mathrm{H}_{2} \mathrm{O}_{2}-0.18 \times \mathrm{Fe}(\mathrm{II}) \times \mathrm{H}_{2} \mathrm{O}_{2}+2.19 \times \mathrm{Fe}(\mathrm{II})^{2}-0.03 \times \mathrm{H}_{2} \mathrm{O}_{2}{ }^{2}
$$

La Tabla 3 resume los valores optimizados para la remoción de carga orgánica contaminante mediante el tratamiento fotocatalítico. La Fig. 1 muestra la superficie de respuesta y los diferentes contornos de la misma. Se presenta una superficie inclinada y pronosticó una posible remoción del orden del $70 \%$ para el proceso optimizado (altos niveles de $\mathrm{H}_{2} \mathrm{O}_{2}$ y bajos niveles de $\mathrm{Fe}(\mathrm{II})$ ).

Tabla 3: Optimización del proceso fotocatalítico homogéneo

\begin{tabular}{|l|c|c|c|}
\hline Factor & Bajo & Alto & Óptimo \\
\hline $\mathrm{Fe}(\mathrm{II}), \mathrm{g} / \mathrm{l}$ & 0.25 & 1.25 & 0.25 \\
\hline $\mathrm{H}_{2} \mathrm{O}_{2} 30 \%, \mathrm{ml}$ & 1.00 & 3.00 & 3.00 \\
\hline \multicolumn{2}{|c|}{ Valor óptimo de remoción COT $=69.22 \%$} \\
\hline
\end{tabular}
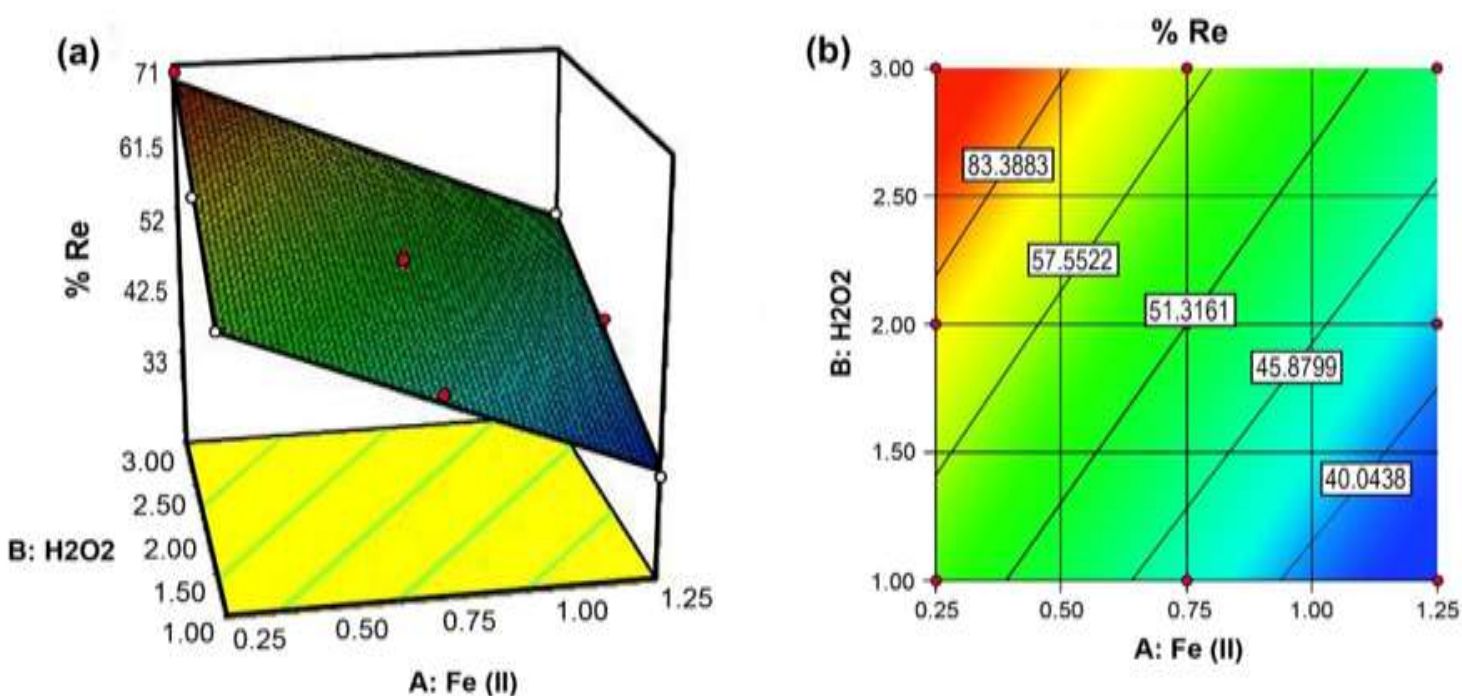

Fig. 1: (a) Superficie de respuesta y (b) líneas de contorno para el tratamiento foto-Fenton del VCC generado durante el procesamiento de subproductos avícolas mediante rendering 
La Fig. 2 muestra las micrografías de la superficie del CA obtenido a partir del material orgánico obtenido como subproducto generado durante el proceso de rendering, con las que se confirmaron tanto la estructura como la porosidad típica de este tipo de material (Rodriguez Reinoso, 2007; Castellar y Garcia, 2011). Los resultados obtenidos de la espectroscopia IR para el CA (Fig. 3), fueron consistentes con las bandas vibracionales reportadas en la literatura (Rodriguez Estupiñan, 2011). En los espectros se observaron cuatro bandas de interés. Una ubicada entre 3200 y $3600 \mathrm{~cm}^{-1}$ asociada a vibraciones de grupos hidroxilo y ácidos carboxílicos, otra entre 2700 y $3000 \mathrm{~cm}^{-1}$ asociada a grupos carboxílicos y carbonilos $(1000-1200 \mathrm{~cm}-1)$ y la última entre 1550 y $1680 \mathrm{~cm}^{-1}$ asociada a quinona.
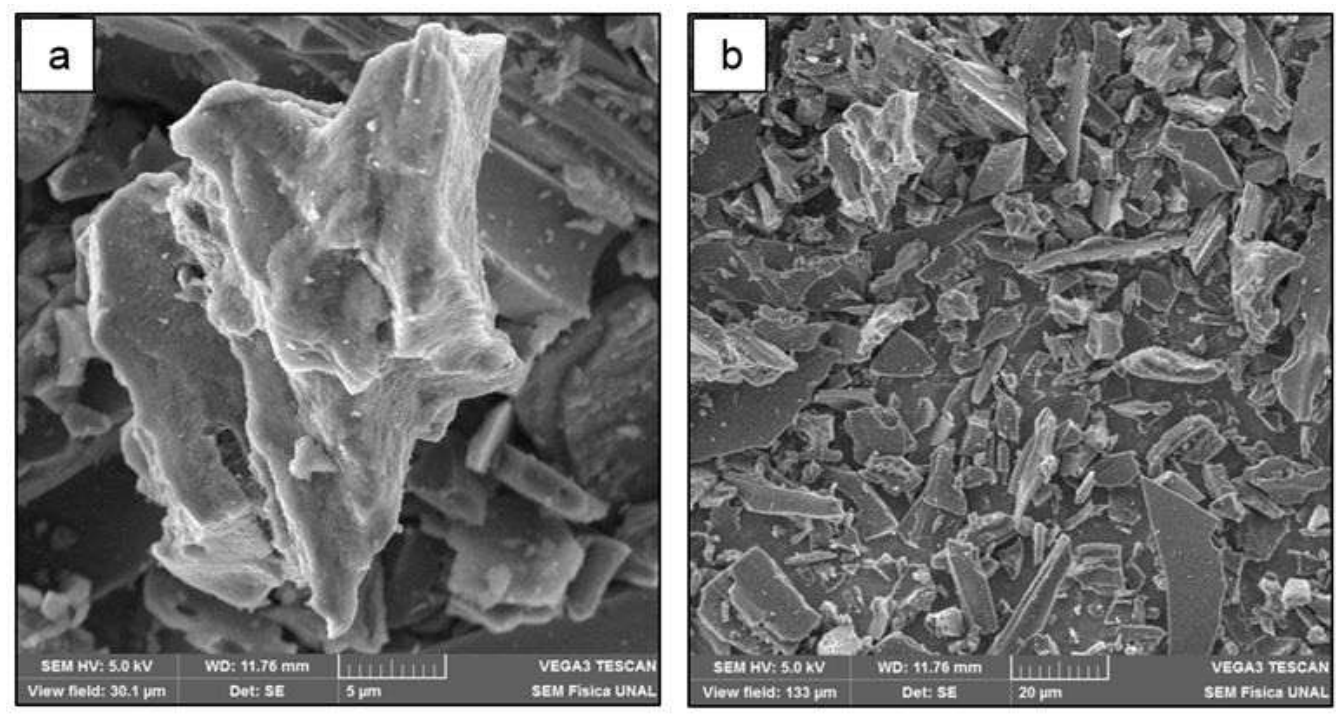

Fig. 2: Micrografía de CA obtenido a partir de subproductos generados en el proceso de rendering. (a) 1000x. (b) 100X

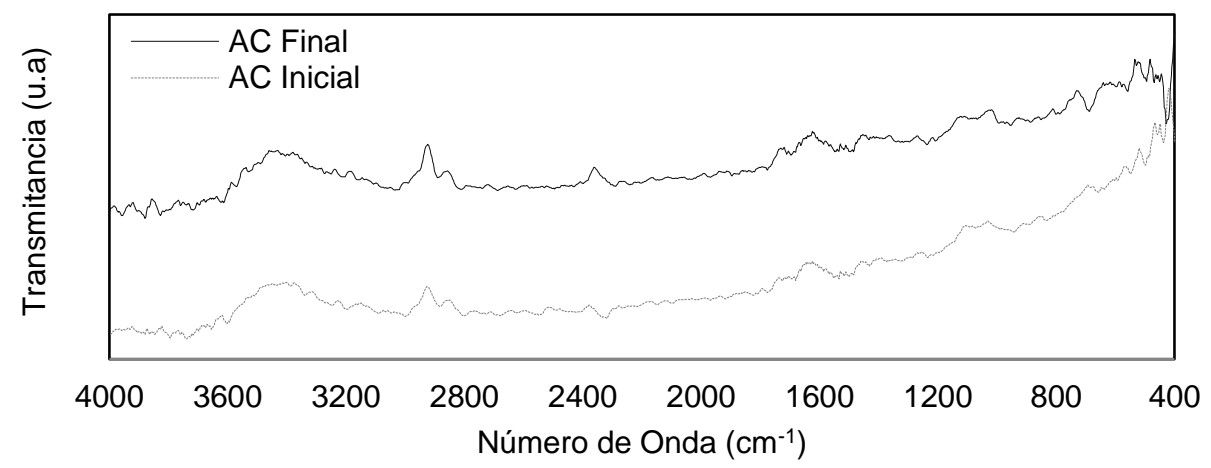

Fig. 3: Espectro FTIR de CA obtenido a partir de subproductos obtenidos del proceso de "rendering"

\section{Adsorción de COT mediante carbón activado (CA)}

Con respecto a los grupos funcionales asociados al fenómeno de adsorción se evidenció un incremento de las señales correspondientes a las bandas presentes entre $3200-3600 \mathrm{~cm}^{-1}$ (asociada a vibraciones de estiramiento $\mathrm{OH}$ en alcoholes y ácidos carboxílicos), $2700-3000 \mathrm{~cm}^{-1}$ (asociada con grupos de ácidos carboxílicos y carbonilos) y en el rango 1000-1200 $\mathrm{cm}^{-1}$ (asociada a grupos ácidos carbonilos). Cabe destacar la aparición de una banda entre 2250-3300 cm-1 asociada al enlace $\mathrm{N}+-\mathrm{H}$ (iones amonio) (Anderson et al., 2004) correspondiente a la adsorción de amoniaco (sustancia presente en el efluente producto de la degradación biológica de las proteínas de los subproductos avícolas) (Sindit, 2006). La Fig. 4 muestra el efecto del pH sobre la adsorción de COT presente en el VCC. La máxima remoción se obtuvo en medio ácido ( 50\%). Por encima de pH 3.0 se observó una disminución en la remoción de la carga contaminante presente en el efluente. La Fig. 5 muestra el efecto de la dosis de adsorbente sobre la remoción de COT presente en el VCC. Un aumento de la dosis de adsorbente incrementó los niveles de remoción del contaminante, sin embargo, concentraciones superiores del mismo no aumentan los niveles de remoción puesto que se alcanza el equilibrio de adsorción (Srivastava y Goyal, 2010). A partir de los resultados obtenidos, se seleccionó una dosis de adsorbente de $2.5 \mathrm{~g}$. A partir de este valor, el porcentaje de remoción de COT fue alrededor del $80 \%$. 


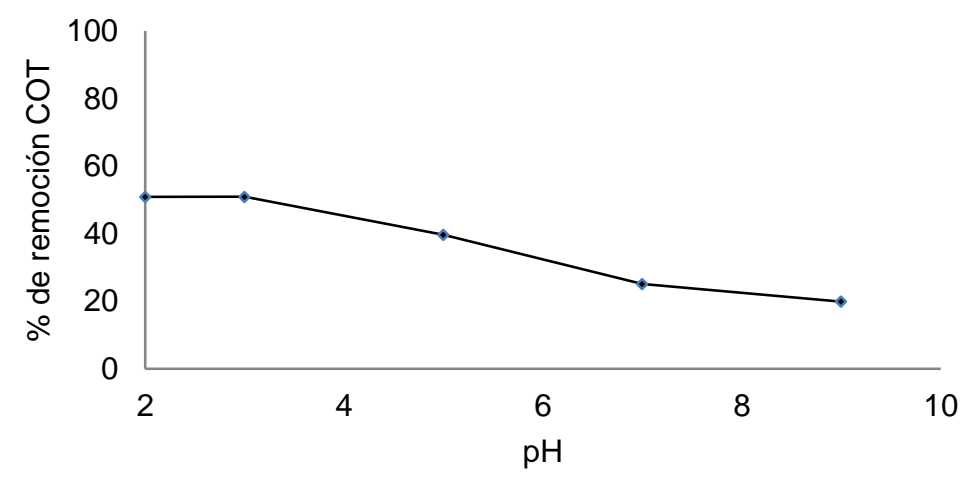

Fig. 4: Efecto del pH sobre el porcentaje de remoción de COT (0,5 g AC, 60 min.)

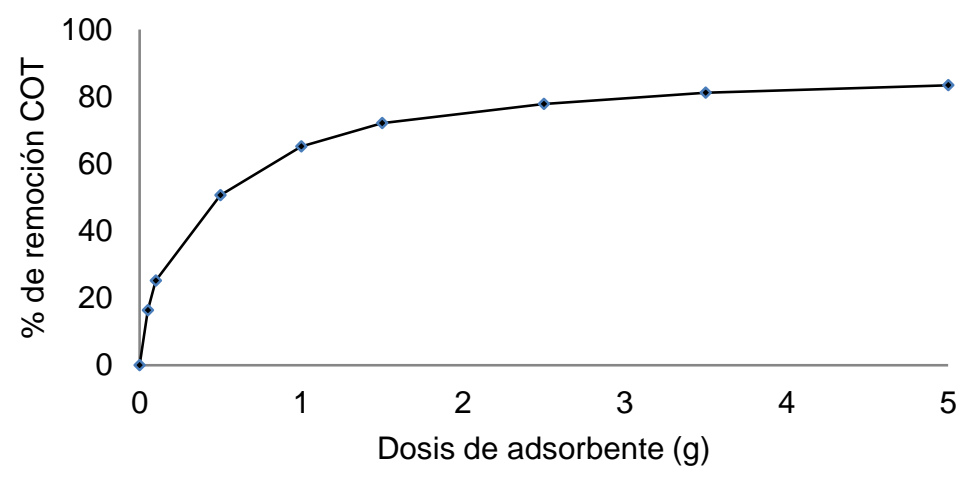

Fig. 5: Efecto de la dosis de adsorbente sobre el porcentaje de remoción de COT mediante AC (pH 3.0, 60 min)

La Fig. 6 muestra el efecto del tiempo sobre la adsorción de COT presente en el VCC. Los elevados niveles de remoción de COT producidos en corto tiempo estuvieron seguidos de una retención gradual hasta alcanzar el equilibrio antes de los 10 minutos de contacto ( 78\% remoción TOC). La Tabla 4 muestra los resultados obtenidos para la determinación del equilibrio de adsorción mediante los modelos de isoterma descritos por Freundlich y Langmuir.

Con base en lo anterior, el modelo de isoterma que más ajustó la adsorción de COT del VCC fue el de Langmuir $\left(R^{2}\right.$ de 0.979$)$ y la capacidad qe fue de $13.59 \mathrm{mg} / \mathrm{g} \mathrm{AC}$. La Tabla 5 muestra el ajuste de los modelos cinéticos. Con base en los resultados, el modelo que mejor ajustó la remoción de COT del VCC después de alcanzado el equilibrio (10 $\mathrm{min})$ fue el pseudosegundo orden $(\mathrm{R} 2=0.9999)$ y el fenómeno que gobernó la adsorción de COT del VCC fue la quimisorción (Ho y McKay, 1999). Para este modelo, la capacidad de adsorción en equilibro $\left(q_{\mathrm{e}}\right)$ fue de $8.99 \mathrm{mg} / \mathrm{g} \mathrm{AC}$.

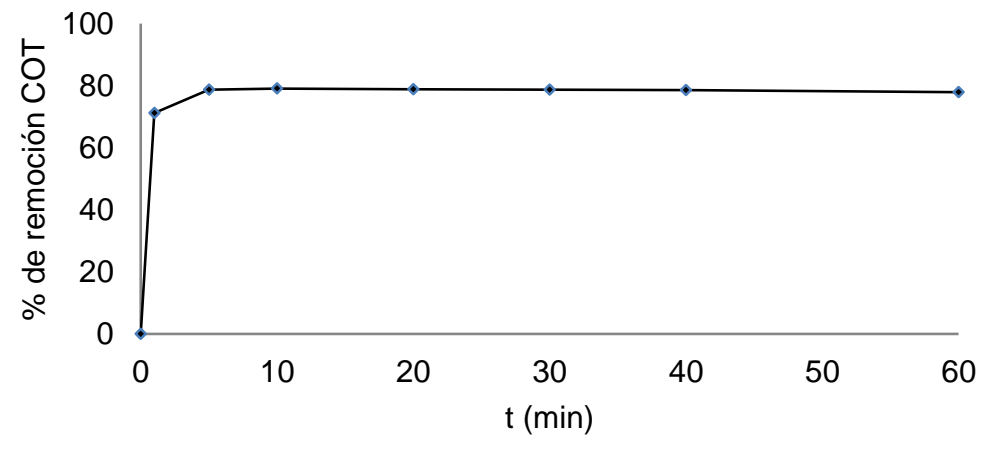

Fig. 6: Efecto del tiempo de contacto sobre el porcentaje de remoción de COT sobre AC (pH 3.0, $2.5 \mathrm{~g} \mathrm{AC)}$ 
Tabla 4: Ecuaciones obtenidas correspondientes a los modelos de isoterma

\begin{tabular}{|c|c|c|c|c|}
\hline Sorbato & Langmuir & $R^{2}$ & Freundlich & $R^{2}$ \\
\hline $\operatorname{COT}(\mathrm{mg} / \mathrm{l})$ & $\mathrm{q}=\frac{0.03 \times 13.59 \mathrm{C}_{\mathrm{e}}}{1+0.03 \mathrm{C}_{\mathrm{e}}}$ & 0.979 & $\mathrm{q}_{\mathrm{e}}=2.43 \times \mathrm{C}_{\mathrm{e}}{ }^{0.31}$ & 0.940 \\
\hline
\end{tabular}

Tabla 5: Constantes para los diferentes modelos cinéticos de adsorción

\begin{tabular}{|c|c|c|}
\hline \multicolumn{2}{|c|}{ Modelo cinético } & $A C$ \\
\hline \multirow{3}{*}{ Pseudo primer orden } & $\mathrm{qe}_{\mathrm{e}}\left(\mathrm{mg} \mathrm{g}^{-1}\right)$ & 1.140 \\
\cline { 2 - 3 } & $\mathrm{k}_{1}\left(\mathrm{~min}^{-1}\right)$ & 0.130 \\
\cline { 2 - 3 } & $\mathrm{R}^{2}$ & 0.647 \\
\hline \multirow{3}{*}{ Pseudo segundo orden } & $\mathrm{qe}_{\mathrm{e}}\left(\mathrm{mg} \mathrm{g}^{-1}\right)$ & 8.990 \\
\cline { 2 - 3 } & $\mathrm{k}_{2}\left(\mathrm{~g} \mathrm{mg}^{-1} \mathrm{~min}^{-1}\right)$ & 0.532 \\
\cline { 2 - 3 } Modelo de orden difuso intraparticular & $\mathrm{R}^{2}$ & 0.999 \\
\cline { 2 - 3 } & $\mathrm{kp}_{\mathrm{p}}\left(\mathrm{mg} \mathrm{g}^{-1} \mathrm{~h}^{-0.5}\right)$ & 0.747 \\
\cline { 2 - 3 } & $\mathrm{C}$ & 4.776 \\
\hline \multirow{2}{*}{ Men } & $\mathrm{R}^{2}$ & 0.647 \\
\hline
\end{tabular}

\section{Acople de los tratamientos de fotocatálisis y adsorción}

Con base en las condiciones óptimas determinadas para el tratamiento fotocatalítico $(0.25 \mathrm{~g} \mathrm{Fe}(\mathrm{II}), 3.0 \mathrm{ml}$ $\mathrm{H}_{2} \mathrm{O}_{2} 30 \% \mathrm{v} / \mathrm{v}$ ) y la adsorción (2.5 g CA, pH 3.0 y 10 min.) la remoción de COT de los sistemas acoplados fotocatálisis/adsorción (1) y adsorción/fotocatálisis (2) se muestran en la Fig. 7.

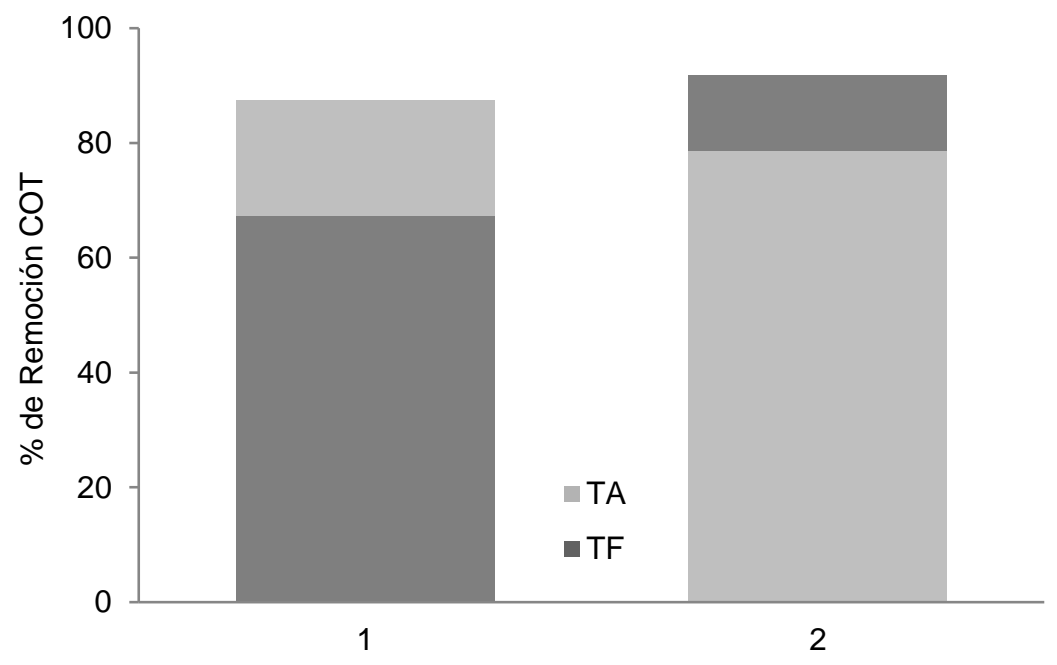

Fig. 7: Remoción de COT presente en el VCC por los sistemas acoplados a pH 3.0. TA (tratamiento de adsorción) y TF (tratamiento fotocatalítico)

Para el sistema de tratamiento 1 , con el pretratamiento fotocatalítico se obtuvo una remoción de COT del $67.39 \%$. Una vez se sometió este efluente al proceso de adsorción con CA, se alcanzó una remoción del $20.01 \%$. Con respecto a la remoción global determinada para el acople, se obtuvo una remoción del contenido de COT del $87.47 \%$. Para el sistema de tratamiento 2, luego del pretratamiento por adsorción se obtuvo una remoción del $78.71 \%$. Este efluente tratado mediante fotocatálisis alcanzó una remoción del 13.1\%. Para acople, la remoción global de COT del VCC fue del 91.92\%. Aunque la mayor remoción se alcanzó mediante el acople 2 (adsorción/fotocatálisis), la diferencia con respecto al tratamiento 1 no fue significativa demostrando que el acople de este tipo de técnicas podría ser considerada dentro de las posibilidades de remediación ambiental para este efluente y se nota en ambas técnicas un efecto sinérgico entre las técnicas de tratamiento.

\section{CONCLUSIONES}

Los resultados del diseño experimental para el tratamiento fotocatalítico homogéneo (foto-Fenton) señalaron que ambas variables (dosis de $\mathrm{Fe}(\mathrm{II})$ y volumen de $\mathrm{H}_{2} \mathrm{O}_{2}$ ) afectan significativamente la remoción de COT. EI equilibrio del proceso sugiere que la adsorción se produce en una superficie homogénea por monocapa en 
la que existen sitios individuales fijos que adsorben igualmente solo una molécula. En el comportamiento cinético se identificó un periodo de adsorción corto, momento a partir del cual es notoria que la concentración del COT tiende a estabilizarse en contacto con el carbón activado. Los datos se ajustaron a un modelo de pseudo segundo orden que demostró que el proceso está gobernado por el fenómeno de quimisorción. Ambas técnicas mostraron aceptables porcentajes de remoción bajo las condiciones evaluadas. El porcentaje de remoción del acople adsorción/fotocatálisis fue mayor con respecto al sistema fotocatálisis/adsorción sin embargo, la diferencia no fue significativa. Con base en la baja biodegradabilidad del efluente, el tratamiento biológico no es una opción viable para la remoción de COT, por lo que la técnica de acople entre estos sistemas podría ser considerada para el tratamiento de este tipo de efluentes.

\section{AGRADECIMIENTOS}

Los autores expresan sus agradecimientos al Convenio 0733 de 2013 entre la Universidad Libre y el Fondo Nacional de Financiamiento para la Ciencia, la Tecnología y la Innovación "Francisco José de Caldas", Convocatoria 617-Modalidad Jóvenes Investigadores e Innovadores de Colciencias.

\section{REFERENCIAS}

Ahmad, Mohd, A., y Alrozi, R. Removal of malachite green dye from aqueous solution using rambutan peelbased activated carbon: Equilibrium, kinetic and thermodynamic studies, doi: 10.1016/j.cej.2011.04.018, Chemical Engineering Journal, (en línea), 171, 510-516 (2011)

Al-Anber, Z., y Mohammed Abu, D. Batch adsorption of cadmium ions from aqueous solution by means of olive cake, doi: 10.1016/j.jhazmat.2007.05.069, Journal of Hazardous Materials, (en línea), 151, 194-201 (2008)

Anderson, R., Bendell, D., y Groundwater, P. Organic spectroscopic analysis, doi: 10.1021/np030773b, Cambridge: The Royal Society of Chemistry, (en línea), (2004)

APHA- AWWA and WPCF. Métodos Normalizados para el análisis de aguas potables y residuales (Primera ed., Vol. I). Madrid, España: Ediciones Díaz de Santos (1992)

Ardila, A., Reyes, J., y Erasmo, V. Degradación fotocatalítica de materia orgánica no biodegradable presente en efluentes de la industria farmacéutica. XXVI Congreso Interamericano de Ingeniería Química. Montevideo: Uruguay (2012)

Awwad, A., y Salem, N. Kinetics and thermodynamics of Cd (II) biosorption onto loquat (Eriobotrya japonica) leaves, doi: 10.1016/j.jscs.2011.10.007, Journal of Saudi Chemical Society, (en línea), 1-8 (2011)

Azouaou, N., Sadaoui, Z., Djaafri, A., y Mokaddem, H. Adsorption of cadmium from aqueous solution onto untreated coffee grounds: Equilibrium, kinetics and thermodynamics, doi: 10.1016/j.jhazmat.2010.08.014, Journal of Hazardous Materials, (en línea), 184, 126-134 (2010)

Castellar, G., y García, A. Remoción de Pb+2 en disolución acuosa sobre carbón activado en polvo: Estudio por lote. Prospect, 9, 59-68 (2011)

Crini, G. Non-conventional low-cost adsorbents for dye removal: A review. doi: 10.1016/j.biortech.2005.05.001, Bioresourse Technology, (en línea), 97, 1061-1085 (2006)

Delfin Pazos, A., Pineda Arellano, C. A., y Silva Martinez, S. Degradación del AEDT y los complejos Cu (II)AEDT y $\mathrm{Cr}$ (III)-AEDT mediante los procesos Fenton y foto-Fenton asistido con radiación solar en soluciones acuosas. Rev. Int. Contam. Ambient, ISSN 0188-4999 (en línea), 4(25), 239-246 (2009) http://www.scielo.org.mx/scielo.php?script=sci_arttext\&pid=S0188-49992009000400004. Acceso: 2015.

Djilani, C., Zaghdoudi, R., Modarressi, A., y Rogalski, M. Elimination of organic micropollutants by adsorption on activated carbon prepared from agricultural waste, doi: 10.1016/j.cej.2012.02.059, Chemical Engineering, (en línea), 189-190, 203-212 (2012)

Gupta, V. Adsorption kinetics and column operations for the removal and recovery of Malachite Green from wastewater using bottom ash, doi: 10.1016/j.seppur.2004.01.008, Separation and purification technology, (en línea), 40, 87-96 (2004) 
Gutiérrez, H., y De la Vara, R. Análisis y diseño de experimentos, $2^{a}$ edición, McGraw-Hill Interamericana, México D.F. (2008)

Ho, Y., y McKay, G. A kinetic study of dye sorption by sorbent waste product pith, doi: 10.1016/S09213449(98)00053-6, Resources, Conservation and Recycling, (en línea), 171-193 (1999)

Kebir, M., Chabani, M., Nasrallah, N., Bensmaili, A., y Trari, M. Coupling adsorption with photocatalysis process for the $\mathrm{Cr}(\mathrm{VI})$ removal, doi: 10.1016/j.desal.2010.11.041, Desalination, (en línea), 270, 166-173 (2011)

Kumar, U., y Bandyopadhyay, M. Sorption of cadmium from aqueous solution using pretreated rice husk, doi:10.1016/j.biortech.2005.02.027, Bioresource Technology, (en línea), 97, 104-109 (2005)

Kumar, J., y Bansal, A. Dual effect of photocatalysis and adsorption in degradation of Azorubine Dye using nanosized TiO2 and actived carbon inmobilized with different techniques. International Journal of ChemTech Research, ISSN : 0974-4290 2(3), 1537-1543 (2010)

Li, Q., Chai, L., y Qin, W. Cadmium (II) adsorption on esterified spend grain: Equilibrium modeling and possible mechanisms, doi: 10.1016/j.cej.2012.04.102, Chemical Engineering Journal, (en línea), 197, 173$180(2012)$

López, A., Almanza, O., Acosta, M., Suarez, A. Degradación Fotocatalítica de Contaminantes Orgánicos Presentes en Agua Residual de la Industria Litográfica mediante Oxido de Bismuto(III)/Oxido de Titanio (Bi2O3/TiO2), doi: 10.4067/S0718-07642016000100016, Información tecnológica, (en línea), 27(1), 147-158 (2016)

Malato, S. Definición de los POAS; fotocatalisis (homogenea y heterogenea), aplicaciones de la luz solar en la fotocatalisis, tecnología de los colectores solares. Plataforma solar de Almería, Ciemat-Ministerio de educación y ciencia, Colombia (2004)

Meeker, D. L. Perspectiva general de la industria del reciclaje de subproductos de origen animal. En t. F. National Renderers Association, y D. L. Meeker (Ed.), Essential Rendering (B. Ruiz, Trad.). Arlington, Virginia, EUA. 306-321 (2006)

Metiver, P. H., Faur, B. C., y Le Cloirec, P. Adsorption of dye onto activated carbon cloths: approach of adsorption mechanism and coupling of ACC with ultrafiltration to treat coloured wastewater, doi: 10.1016/S1383-5866(02)00147-8, Separation and Purification Technology, (en línea), 31, 3-11 (2003)

Ministerio de Ambiente y Desarrollo Sostenible. Guía Ambiental para el Subsector Avícola. Bogotá D.C., Colombia: Ministerio de Ambiente y Desarrollo Sostenible (2013)

Ministerio de Ambiente y Desarrollo Sostenible. Resolución 0631 (17 Marzo de 2015). Por la cual se establecen los parámetros y los valores límites máximos permisibles en los vertimientos puntuales a cuerpos de aguas superficiales y a los sistemas de alcantarillado público y se dictan otras disposiciones. Colombia (2015)

Montgomery, D. Diseño y análisis de experimentos, $2^{a}$ edición, Limusa Wiley, México D.F (2004)

Moreno, H., Droppelmann, C., y Verdejo, M. Evaluación de Carbón Activado Producido a partir de Lodo Generado en una Planta de Tratamiento de Aguas Servidas, doi: 10.4067/S0718-07642006000300003, Información Tecnológica, (en línea), 17(3), 9-14 (2006)

Moreno, $\mathrm{J}$ y Giraldo, L. Propiedades Termodinámicas del Proceso de Adsorción de $\mathrm{Pb}^{2+}$ sobre Carbón Activado a Diferentes pH, doi: 10.1612/inf.tecnol.3923it.07, Información Tecnológica, (en línea), 19(5), 61-72 (2008)

Özer, A., y Pirincci, $\mathrm{H}$. The adsorption of $\mathrm{Cd}(\mathrm{II})$ ions on sulphiric acid-treated wheat bran, doi:10.1016/j.jhazmat.2006.03.009, Journal of Hazardous Materials, (en línea), 137, 849-855 (2006)

Rodríguez Estupiñan, J. Modificación y caracteización calorimétrica de carbón activado granular, para la remoción de Cd (II) y Ni (II) en Adsorción Simple y Competitiva (Trabajo de investigación, Magister en Ciencias-Química). Bogotá D.C.: Universidad Nacional de Colombia (2011) 
Rodríguez Reinoso, F. El carbón activado como adsorbente universal. En J. C. Moreno Pirajan, Sólidos porosos: Preparación, caracterización y aplicaciones. Bogotá D.C., Colombia: Universidad de los Andes, 3-4 (2007)

Sayed, S. A., Saleh, S. M., y Hasan, E. E. Removal of some polluting metals from industrial water using chicken feathers, doi:10.1016/j.desal.2005.02.022, Desalination, (en línea), 182, 243-255 (2005)

Shon, H. K., Vigneswaran, S., Ngo, H. H., y Kim, J. H. Chemical coupling of photocatalysis with flocculation and adsorption in the removal of organic matter, doi:10.1016/j.watres.2005.04.066, Water Research, (en línea), 39, 2549-2558 (2005)

Sindit, G. Environmental Issues in the Rendering Industry. In T. F. NATIONAL RENDERERS ASSOCIATION, y D. Meeker (Ed.), Essential Rendering (pp. 311-312). Arlington, Virginia, EUA: National Renderers Association, the Fats and Proteins Research Foundation (FPRF), and the Animal Protein Producers Industry (APPI), 311-312 (2006)

Srivastava, S., y Goyal, P. Novel Biomaterials, Environmental Science and Engineering. New York: SpringerVerlag Heidelberg (2010)

Thinakaran, N. Equilibrium and Kinetic studies on the removal of Acid Red 114 from aqueous solutions using activated carbons prepared from seed shells, doi:10.1016/j.jhazmat.2008.01.043, Journal of Hazardous Materials, (en línea), 158, 142-150 (2008)

Wei, Wei-Ling, S., Yan-Zhi, Q., Qing, Y., y Jin-ren, N. Adsorption of organic pollutants from coking and papermaking wastewaters by bottom ash, doi: 10.1016/j.jhazmat.2007.10.063, Journal of Hazardous Materials, (en línea), 154, 595-601 (2008)

Yank, R. Adsorbents: Fundamentals and applications. Hoboken, New Jersey: John Wiley y Sons (2003)

Zhang, X., Hu, A., Luong, D., Oakes, K., Servos, M., Liang, R., Zhang, H. A. Adsorption and photocatalytic degradation kinetics of pharmaceuticals by TiO2 nanowires during water treatment, doi:10.1007/s12649-0129142-6, Waste Biomass Valor, (en línea), (2012) 